\title{
Complete Genome Sequence and Evaluation of Expression Level for Genes Associated with Virulence for a Clinical Brucella Melitensis Strain C-573 Isolated in Russia
}

\author{
Dmitry A. Kovalev, Sergey V. Pisarenko", Anna Yu. Evchenko, Dmitry G. Ponomarenko, Olga V. \\ Bobrysheva, Andrei M. Zhirov, Irina V. Kuznetsova, Diana V. Rusanova, Alexander N. Kulichenko \\ Laboratory of Biochemistry, Stavropol Research Anti-Plague Institute, 355035, Stavropol, Russia \\ Email: pisarenko_sv@mail.ru
}

\begin{abstract}
Brucellosis is one of the most pressing global zoonotic diseases, which is endemic in many regions of the world. It is believed that Brucella melitensis is the most pathogenic species of Brucella genus for humans. However, the processes underlying the pathogenicity of this pathogen remain not fully understood. In our study, we report on the first complete genome of the clinical B. melitensis strain isolated in Russia, perform structural and functional analysis of the genomic sequence, and evaluate the expression level of genes associated with virulence based on Next Generation Sequencing (NGS) data. The obtained information on the genetic similarities and differences between $B$. melitensis strains can be used to study the mechanisms responsible for the pathogenicity of Brucella spp., as well as in the process of developing new therapeutic and preventive strategies for controlling brucellosis.
\end{abstract}

Keywords: Brucella melitensis, complete genome, NGS, gene expression

\section{Introduction}

Brucellosis is a particularly dangerous zoonotic disease, which in the acute form typically presents with high, undulating fever, while chronic brucellosis may affect many host organs ${ }^{[1]}$. Brucella classification is based on the host preference of the pathogen and its phenotypic and biochemical properties ${ }^{[2-3]}$.

B. melitensis and B. abortus cause most serious forms of disease, and therefore their epidemiological significance is quite high ${ }^{[4]}$. Human infection occurs via alimentary, contact or air-dust mode of transmission when human contacts infected animals or consumes products of animal origin, especially unpasteurized milk products contaminated with Brucella spp. ${ }^{[5]}$.

Nowadays brucellosis remains an urgent problem for the whole world with 500,000 cases reported annually. The highest incidence of brucellosis is registered in the countries of South America, Africa, the Middle East and most of Asia ${ }^{[6-7]}$. In Russia, the epidemic situation remains unstable: while the number of cases of human brucellosis is decreased compared to that of previous years, the epizootic situation in livestock exacerbates. The most endemic regions, mostly due to the high development of agriculture, are North Caucasus, Southern, Siberian and Volga Federal districts. According to the data of the Reference center that monitoring the causative agent of brucellosis (Stavropol Research Anti-Plague Institute), 90\% of all cases registered in Russia occur on these territories ${ }^{[8]}$. At that, most cases of human infection are associated with $B$. melitensis.

The Brucella spp. lack classic virulence factors ${ }^{[9]}$; however, these bacteria are able to adapt to various environmental conditions, and their virulence is determined by their ability to survive and reproduce in the macrophages of the host organism. The key role in the pathogenesis process belongs to the genes involved in survival processes in host macrophages. Although these factors are only indirectly associated with clinical manifestations of brucellosis, they are crucial for surviving and reproducing inside the host cells. Thus, the accumulation of data on the structure and functional features of the genomes of Brucella strains is an important step in the process of studying the mechanisms that determine their pathogenicity.

There is very little published information in international databases about the Brucella spp. strains isolated on the territory of Russia. The available data on the genomic sequences of Russian strains are presented in the form of fragmented

Copyright (C2020 Sergey V. Pisarenko, et al

DOI: https://doi.org/10.37256/amtt.122020626

This is an open-access article distributed under a CC BY license

(Creative Commons Attribution 4.0 International License)

https://creativecommons.org/licenses/by/4.0/ 
draft assemblies, while only complete genome assemblies may provide full information on the structure of the genome and its functional features.

In the previous research ${ }^{[10]}$, we were able to establish the phylogenetic relationship of B. melitensis strain C-573 with other species of Brucella genus. Further research revealed that B. melitensis strain C-573 has physiological, morphological and biochemical properties typical for strains isolated in the southern part of European Russia. In the current paper, we provide a complete genomic sequence of a clinical isolate B. melitensis C-573, isolated from human in 2014, describe structural and functional features of the genome and evaluate the level of expression of genes associated with virulence. New information on the genetic similarities and differences between B. melitensis strains, in addition to studying the processes causing pathogenicity of Brucella spp., can be used in the development of antimicrobial agents and vaccines by identifying common conservative targets for all strains of the species.

\section{Materials and methods}

\subsection{Bacterial strain}

B. melitensis strain C-573 used in this study was isolated from a person diagnosed with brucellosis in the Stavropol Territory in 2014. The culture was obtained through a direct inoculation of mammary gland punctate to a biphasic medium (Castaneda method). The strain was identified via standard biochemical methods in accordance with "Procedure for organizing and conducting laboratory diagnostics of brucellosis for laboratories at the territorial, regional and federal levels" (MUK 4.2.3010-12). Morphological and biochemical properties of the strain state that this strain belongs to B. melitensis biovar II. According to antimicrobial susceptibility testing results, the strain is sensitive to gentamicin, amikacin, kanamycin, streptomycin, doxycycline, tetracycline, nalidixic acid, ciprofloxacin, ofloxacin, pefloxacin, levofloxacin, and rifampicin. Antimicrobial susceptibility testing was performed using disc diffusion method with "Set of discs for antimicrobial susceptibility testing-1" (Institute Pasteur in Saint-Petersburg for Research in Epidemiology and Microbiology of Federal Service for Surveillance on Consumer Rights Protection and Human Wellbeing) in accordance with manufacturer's instructions.

Bacterial strain B. melitensis C-573 used in this study, was obtained from the State Collection of Pathogenic Microorganisms of Stavropol Research Anti-Plague Institute, where it was stored in a lyophilized state.

\subsection{Preparation of nucleic acid samples}

Bacteria were cultivated on Brucella Agar at $37^{\circ} \mathrm{C}$ for 48 hours. The microbial suspension of $2 \times 10^{9} \mathrm{~m} . \mathrm{sub} / \mathrm{ml}$ was decontaminated by adding thiomersal to a final concentration of $0.01 \%$ and subsequent incubation at $56^{\circ} \mathrm{C}$ for 30 minutes; after that sterile DNA was isolated. Genomic DNA was isolated from $0.5 \mathrm{ml}$ of decontaminated microbial suspension with the use of PureLink Genomic DNA Kits (Life Technologies, USA). RNA was isolated using $1 \mathrm{ml}$ of microbial suspension with concentration of $2 \times 10^{9} \mathrm{mc} / \mathrm{ml}$ and TRIzol Max Bacterial RNA Isolation Kit (Life Technologies, USA); manipulations were performed as described in the manufacturer's protocol.

Briefly, after centrifugation, the microbial suspension precipitate was resuspended in $200 \mu \mathrm{l}$ of pre-warmed TRIzol Reagent MAX to $95^{\circ} \mathrm{C}$ and incubated at $95^{\circ} \mathrm{C}$ for $4 \mathrm{~min}$. Then, $1 \mathrm{ml}$ of TRIzol Reagent was added to the suspension and incubated for $5 \mathrm{~min}$ at room temperature, after which $200 \mu \mathrm{l}$ of chloroform was added and incubated for another 2-3 min. After centrifugation for $15 \mathrm{~min}$ at $12000 \mathrm{~g}$ and $4^{\circ} \mathrm{C}$, the aqueous (transparent) phase was collected. RNA was precipitated with $100 \%$ isopropanol and $1 \mathrm{ml}$ of $75 \%$ ethanol. The finished RNA sample was dissolved in $50 \mu \mathrm{l}$ of RNase-free water and stored at $-80^{\circ} \mathrm{C}$.

The concentration of nucleic acids was determined using Qubit dsDNA HS Assay Kit and Qubit PHK HS Assay Kit (Invitrogen, Life Technologies, USA). The purity of the genomic DNA was evaluated with a NanoDrop 2000 spectrophotometer (Thermo Scientific, USA). In addition, the size distribution of RNA fragments was evaluated using a reagent kit for visualizing fragments of the RNA library: Experion RNA Analysis Kit and Experion ${ }^{\mathrm{TM}}$ Automated Electrophoresis System. Sample preparation was carried out in the Laboratory of Brucellosis Research of the Stavropol AntiPlague Institute of Rospotrebnadzor.

\subsection{Genome and transcriptome sequencing}

Preparation of DNA libraries with a read length of $400 \mathrm{bp}$. was performed with the use of Ion XpressTM Plus Fragment Library Kit (Life Technologies, USA) in accordance with the manufacturer's protocol. DNA library fragments were separated with the use of 2\% E-Gel SizeSelect agarose gel (Invitrogen, USA). The finished libraries were purified with the use of Agencourt AMPure XP magnetic particles (Beckman Coulter, USA). Libraries quality and concentration were determined using the Experion ${ }^{\mathrm{TM}}$ Automated Electrophoresis System, Experion DNA 1K Reagents and Supplies and 
Experion DNA Chips kits (Bio-Rad, USA).

For the RNA library preparation, we have used a sample with RNA Quality Indicator (RQI) $>9$. rRNA was removed from the RNA sample with the use of RiboMinus ${ }^{\mathrm{TM}}$ Transcriptome Isolation Kit bacteria (Life Technologies, USA) as described in the manufacturer's protocol. Quality and quantity of the RNA after the removal of rRNA was evaluated with the use of Experion ${ }^{\mathrm{TM}}$ Automated Electrophoresis System and Experion RNA Analysis Kit. cDNA library was prepared using Ion Total RNA-Seq kit v2 in accordance with the manufacturer's protocol. After evaluating fragment size distribution and quantitative analysis of the concentration of cDNA library, we have performed monoclonal amplification on microspheres.

Monoclonal amplification on microspheres was performed using Ion OneTouch 400 Template Kit (Life Technologies, USA) according to the manufacturer's protocol. Microspheres were enriched with Dynabeads MyOne Streptavidin C1 magnetic particles (Invitrogen, Life Technologies, USA). The efficiency of the enrichment process was evaluated using Ion Sphere Quality Control Kit (Life Technologies, USA). Nucleic acid sequencing was performed using Ion Torrent PGM sequencer and Ion 318 Chips Kit V2 (Life Technologies, USA).

\subsection{Post-sequencing data processing}

Evaluation of the quality of the obtained data was carried out using the FastQC program version $0.11 .3^{[11]}$. Reads containing nucleotides with quality of reading, $\mathrm{Q}<15$ were removed in Trimmomatic version $0.33^{[12]}$. Reads with an average quality of $\mathrm{Q}<20$, as well as reads less than 75 nucleotides long were removed.

\subsection{Genome de novo assembly}

Genome de novo assembly was performed via Newbler v 3.0 (Roche, Switzerland); the extent of the genome coverage was more than $80 \times$. To finish the complete assembly, we have performed mapping of the contigs obtained during de novo assembly on reference genome of B. melitensis $16 \mathrm{M}$ (GeneBank: NC_003317.1, NC_003318.1) in Mauve v. 2.4.0 software ${ }^{[13]}$. After that, primers complementary to the ends of each contig were selected so that during PCR the sections corresponding to gaps in the draft assembly were amplified. The same DNA sample was used as a template for PCR as was used in the preparation of fragment libraries for genome sequencing. The obtained DNA fragments were sequenced by Sanger method using an Applied Biosystems 3500 Genetic Analyzer sequencer (Applied Biosystems, USA). The process of assembly, which consists of aligning and merging of the contigs and DNA fragments obtained during Sanger sequencing, was performed in Contig Express app from Vector NTI Suite software package ${ }^{[14]}$.

\subsection{Annotation and analysis of orthologous gene clusters}

Genome annotation was performed using NCBI Prokaryotic Genome Annotation Pipeline ${ }^{[15]}$. An analysis of the distribution of overlapping orthologous gene clusters was performed using OrthoVenn web interface ${ }^{[16]}$.

\subsection{Gene expression level analysis}

We used HISAT2 software to map the reads to the reference genome ${ }^{[17]}$. HTSeq-count v. 0.6 .1 was used to count readings mapped to coding regions of the genome ${ }^{[18]}$. Mapping results were normalized to compare gene coverage values by calculating the RPKM (Reads per kilobase per million mapped reads) values for each gene ${ }^{[19]}$.

\section{Results and discussion}

\subsection{Structural features of the genome of the $B$. melitensis $\mathbf{C}-573$ strain}

The genome of B. melitensis C-573 is a plasmid-free genome with two circular chomosomes of 2,125,194 and $1,185,595 \mathrm{bp}$. The total length is $3,310,789 \mathrm{bp}$, and the $\mathrm{G}+\mathrm{C}$ content of the assembly corresponds to a species-specific value of $57.22 \%$. Of the total 3245 genes, 3168 are protein coding genes, 77 encode RNA and 217 appeared to be pseudogenes. For 2676 (82.47\%) genes, functions were predicted and 2427 genes could be assigned to a COG functional category. The genome statistics are provided in Table 1 and Figure 1. 
Table 1. Genome statistics

\begin{tabular}{ccc}
\hline Attribute & Value & \% of Total \\
\hline Genome size (bp) & 3310789 & 100 \\
DNA coding (bp) & 2874326 & 86.82 \\
DNA G + C (bp) & 1894445 & 57.22 \\
DNA scaffolds & 2 & 100 \\
Total genes & 3245 & 97.63 \\
Protein coding genes & 3168 & 91.60 \\
RNA genes & 77 & 2.37 \\
Pseudo genes & 217 & 6.4 \\
Genes in internal clusters & NA & NA \\
Genes with function prediction & 2676 & 82.47 \\
Genes assigned to COGs & 2427 & 74.79 \\
Genes with Pfam domains & 2756 & 84.93 \\
Genes with signal peptides & 273 & 8.41 \\
Genes with transmembrane helices & 799 & 24.62 \\
CRISPR repeats & NA & NA \\
\hline
\end{tabular}
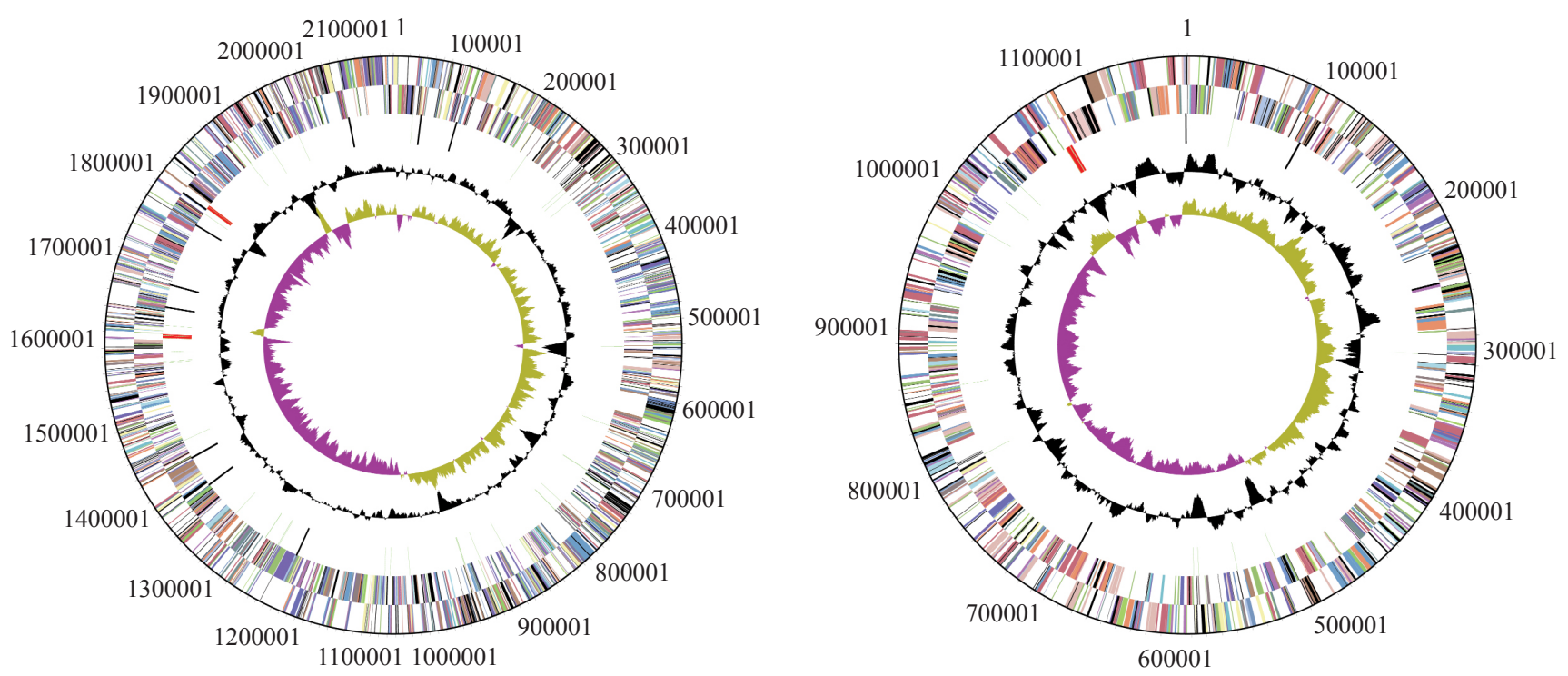

\begin{tabular}{|clll|}
\hline COG Code & \multicolumn{1}{c|}{ COG Function Definition } & COG Code & COG Function Definition \\
{$[\mathrm{A}]$} & RNA processing and modification & {$[\mathrm{O}]$} & Posttranslational modification, protein turnover, chaperones \\
{$[\mathrm{B}]$} & Chromatin structure and dynamics & {$[\mathrm{P}]$} & Inorganic ion transport and metabolism \\
{$[\mathrm{C}]$} & Energy production and conversion & {$[\mathrm{Q}]$} & Secondary metabolites biosynthesis, transport and catabolism \\
{$[\mathrm{D}]$} & Cell cycle control, cell division, chromosome partitioning & {$[\mathrm{R}]$} & General function prediction only \\
{$[\mathrm{E}]$} & Amino acid transport and metabolism & {$[\mathrm{S}]$} & Function unknown \\
{$[\mathrm{F}]$} & Nucleotide transport and metabolism & {$[\mathrm{T}]$} & Signal transduction mechanisms \\
{$[\mathrm{G}]$} & Carbohydrate transport and metabolism & {$[\mathrm{U}]$} & Intracellular trafficking, secretion, and vesicular transport \\
{$[\mathrm{H}]$} & Coenzyme transport and metabolism & {$[\mathrm{V}]$} & Defense mechanisms \\
{$[[\mathrm{I}]$} & Lipid transport and metabolism & {$[\mathrm{W}]$} & Extracellular structures \\
{$[\mathrm{J}]$} & Translation, ribosomal structure and biogenesis & {$[\mathrm{X}]$} & Mobilome: prophages, transposons \\
{$[\mathrm{K}]$} & Transcription & {$[\mathrm{Y}]$} & Nuclear structure \\
{$[\mathrm{L}]$} & Replication, recombination and repair & {$[\mathrm{Z}]$} & Cytoskeleton \\
{$[\mathrm{M}]$} & Cell wall/membrane/envelope biogenesis & {$[\mathrm{NA}]$} & Not Assigned \\
\hline $\mathrm{N}]$ & Cell motility & & \\
\end{tabular}

Figure 1. Circular map of two chromosomes

From outside to the center: Genes on forward strand (colored by COG categories), Genes on reverse strand (colored by COG categories), RNA genes (tRNAs green, rRNAs red, other RNAs black), GC content, GC skew

Functional annotations were predicted for 2676 coding sequences, which is $82.47 \%$ of the total number of genes. 2427 coding sequences ( $74 \%$ of the total number of genes) were assigned to the functional categories of $\mathrm{COG}$; their distribution by functional groups is presented in Table 2 . 
Table 2. Distribution of genes by functional categories of COG

\begin{tabular}{|c|c|c|c|}
\hline COG Code & COG Function Definition & Count & Percent \\
\hline$[\mathrm{A}]$ & RNA processing and modification & NA & NA \\
\hline [B] & Chromatin structure and dynamics & 1 & 0.04 \\
\hline$[\mathrm{C}]$ & Energy production and conversion & 171 & 6.34 \\
\hline [D] & Cell cycle control, cell division, chromosome partitioning & 30 & 1.11 \\
\hline [E] & Amino acid transport and metabolism & 308 & 11.42 \\
\hline$[\mathrm{F}]$ & Nucleotide transport and metabolism & 75 & 2.78 \\
\hline [G] & Carbohydrate transport and metabolism & 180 & 6.68 \\
\hline$[\mathrm{H}]$ & Coenzyme transport and metabolism & 150 & 5.56 \\
\hline$[\mathrm{I}]$ & Lipid transport and metabolism & 129 & 4.78 \\
\hline$[\mathrm{J}]$ & Translation, ribosomal structure and biogenesis & 185 & 6.86 \\
\hline$[\mathrm{K}]$ & Transcription & 174 & 6.45 \\
\hline$[\mathrm{L}]$ & Replication, recombination and repair & 93 & 3.45 \\
\hline$[\mathrm{M}]$ & Cell wall/membrane/envelope biogenesis & 175 & 6.49 \\
\hline$[\mathrm{N}]$ & Cell motility & 31 & 1.15 \\
\hline$[\mathrm{O}]$ & Posttranslational modification, protein turnover, chaperones & 126 & 4.67 \\
\hline$[\mathrm{P}]$ & Inorganic ion transport and metabolism & 160 & 5.93 \\
\hline [Q] & Secondary metabolites biosynthesis, transport and catabolism & 70 & 2.60 \\
\hline$[\mathrm{R}]$ & General function prediction only & 224 & 8.31 \\
\hline$[\mathrm{S}]$ & Function unknown & 184 & 6.82 \\
\hline$[\mathrm{T}]$ & Signal transduction mechanisms & 94 & 3.49 \\
\hline$[\mathrm{U}]$ & Intracellular trafficking, secretion, and vesicular transport & 39 & 1.45 \\
\hline$[\mathrm{V}]$ & Defense mechanisms & 59 & 2.19 \\
\hline$[\mathrm{W}]$ & Extracellular structures & 3 & 0.11 \\
\hline$[\mathrm{X}]$ & Mobilome: prophages, transposons & 35 & 1.30 \\
\hline$[\mathrm{Y}]$ & Nuclear structure & NA & NA \\
\hline$[\mathrm{Z}]$ & Cytoskeleton & NA & NA \\
\hline [NA] & Not Assigned & NA & NA \\
\hline
\end{tabular}

The genomic sequence of the $B$. melitensis $\mathrm{C}-573$ isolate is $99.9 \%$ similar to the genome of the reference $B$. melitensis strain 16 M (GeneBank: NC_003317.1, NC_003318.1). When comparing the genomes of B. melitensis C-573 and B. melitensis $16 \mathrm{M}$ strains, we have detected genomic rearrangements in both chromosomes. In the first chromosome, we have found two insertions, while one insertion and one inversion were found on the second.

$3952 \mathrm{bp}$ insertion in the first chromosome (555133-559085, the coordinates of the genomic rearrangements are given in accordance with the complete assembly of the genome of the B. melitensis C-573 strain) includes the genes of the prokaryotic DNA-binding protein, which belongs to the family of transcriptional regulators of xenobiotic response elements, the amino acid ABC transporter, AzID protein and transposase IS2020 from the family of transposases IS6. The genes in the structure of this insertion are involved in the processes of branched-chain amino acid transport.

The second insertion (3538 bp, 1481462-1485000) on this chromosome contains the coding sequences of the phage main capsid protein, hypothetical protein, adapter protein, DUF3168 domain-containing protein. The main phage capsid protein is species-specific protein for B. melitensis, the remaining listed proteins are found in different species of the Brucella genus. The listed genes encode proteins involved in the processes that provide the cell's resistance to bacterial pathogens.

$6815 \mathrm{bp}$ insertion on the second chromosome (590980-597795) includes the genes of FAD-binding oxidoreductase, a hypothetical protein, sugar-dehydrogenase, a transcription regulator of the Lrp/AsnC family, amidohydrolase, and 3-oxoadipyl-CoA thiolase. The encoded proteins are involved in the formation of cellular response to exogenous amino acid effectors by regulating lysine metabolism, amino acid transport, transcription activation of oxygen for oxidation and hydroxylation processes.

Inverted region of the second chromosome with a length of $45656 \mathrm{bp}$ (1035284-1080940) includes 42 genes, among them are genes encoding transport proteins, proteins of the dehydrogenase family, transferases, transcriptional regulators, penicillin acylases, hypothetical proteins, the PemK/MazF toxin-antitoxin system gene, and others. Functional annotation of all genes located in the regions of genomic rearrangements are presented in Table 3. 
Table 3. Functional annotation of all genes located in the regions of genomic rearrangements

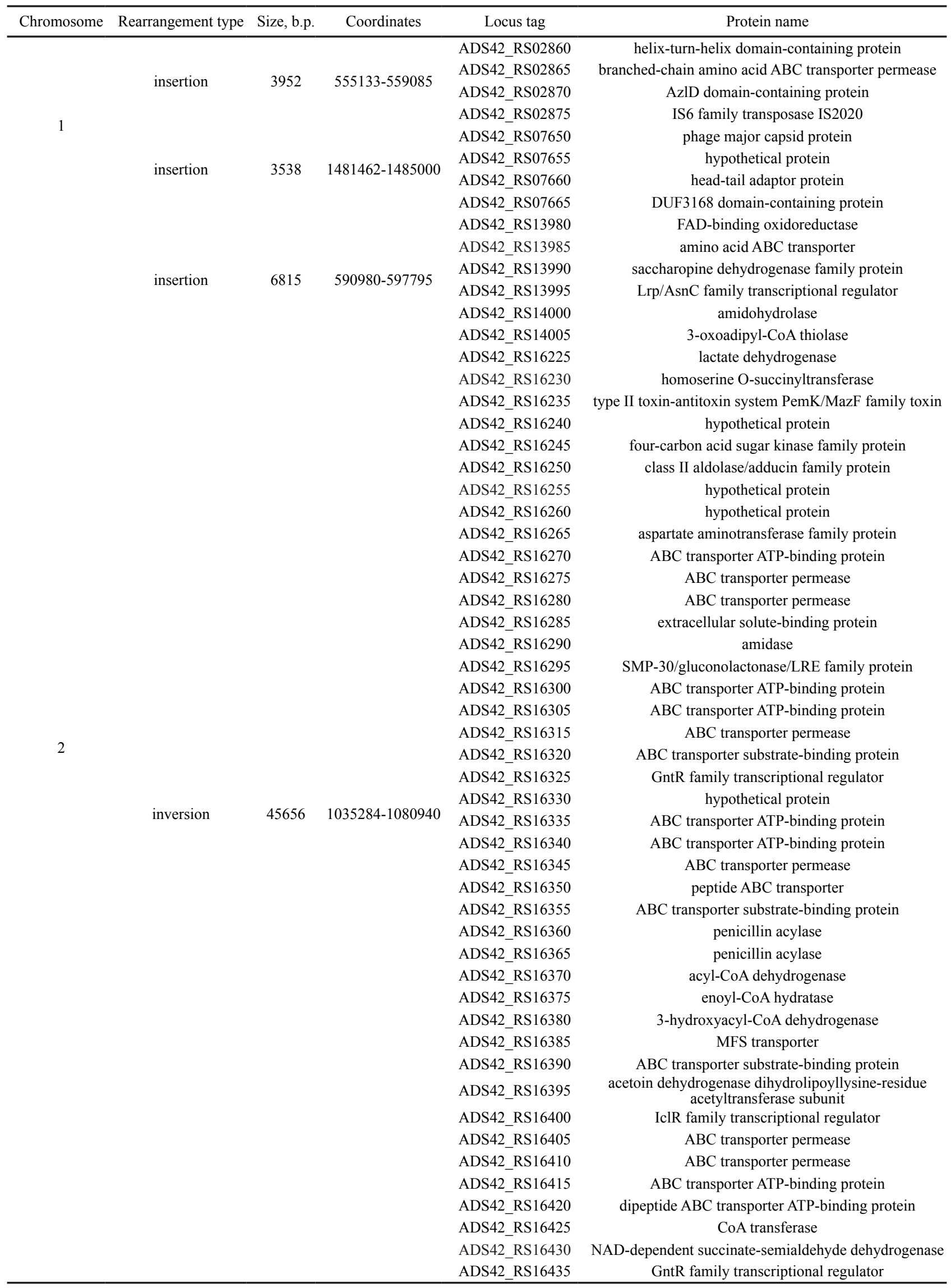




\subsection{Functional features of the genome of $\boldsymbol{B}$. melitensis strain $\mathbf{C}-573$}

To describe the functional features of the genome of the B. melitensis $\mathrm{C}-573$ strain, we have carried out a comparative analysis of the genes of four strains: B. melitensis $16 \mathrm{M}$ (GeneBank ID: GCA_000007125.1), B. melitensis Ether (GeneBank ID: GCA_000740355.1), B. melitensis ATCC 23457 (GeneBank ID: GCA_000022625.1) and B. melitensis C-573. The strains selected for comparison belong to different genotypes established during the phylogenetic analysis performed earlier $^{[10]}$. B. melitensis strain Ether belongs to the first genotype, B. melitensis strain ATCC 23457 to the second, and $B$. melitensis strain $16 \mathrm{M}$ to the fifth genotype. All genomes selected from the international GenBank database for analysis were presented as complete assemblies. We did not use fragmented genome assemblies for the analysis as using them could have caused erroneous results. At the time of the analysis, complete assemblies for third and fourth genotype did not exist, so we have excluded these genotypes from the analysis. Results are presented in Figure 2.

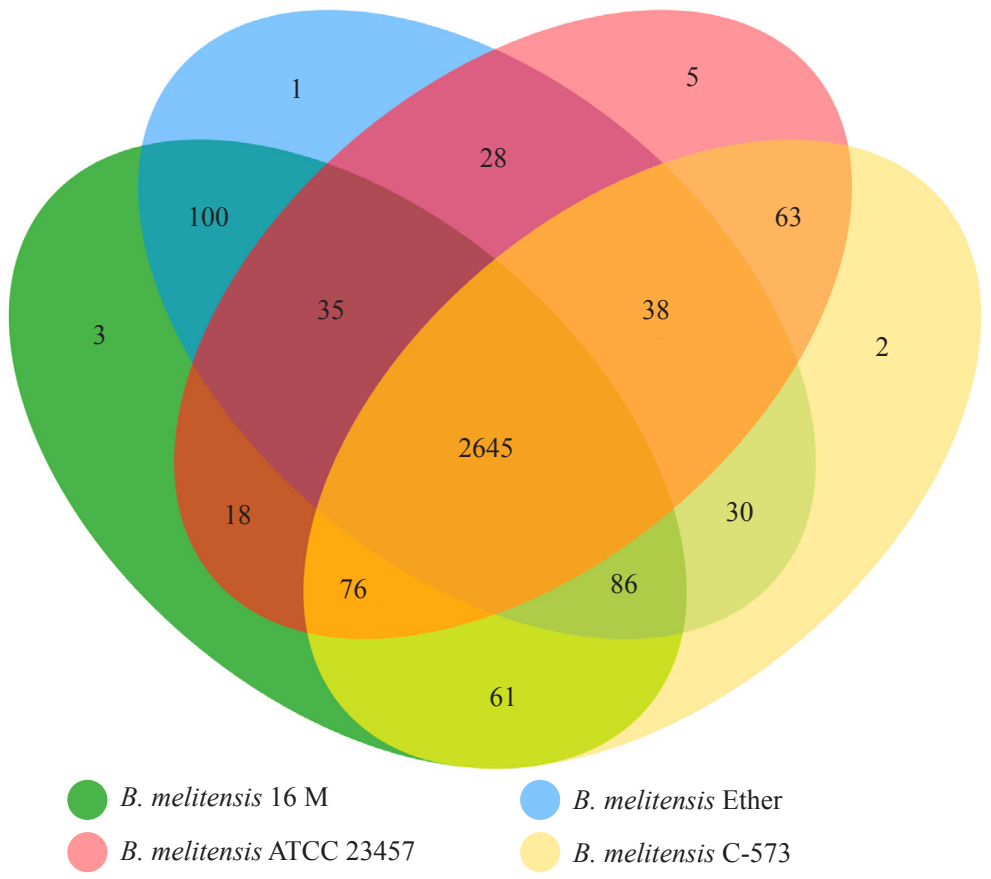

Figure 2. Distribution of overlapping orthologous gene clusters

2645 gene clusters are common to all four strains, 86 gene clusters are common to B. melitensis $16 \mathrm{M}$, B. melitensis Ether and B. melitensis C-573 strains, 76 clusters are common to B. melitensis $16 \mathrm{M}$, B. melitensis ATCC 23457 and B. melitensis C-573, another 38 gene clusters are common for B. melitensis ATCC 23457, B. melitensis Ether and B. melitensis $\mathrm{C}-573$ strains. In the pairwise comparison of common gene clusters, we have detected the smallest number of common clusters in B. melitensis C-573 and B. melitensis Ether strains-30 clusters. Comparison of genes of $B$. melitensis C-573 and B. melitensis $16 \mathrm{M}$ strains revealed 61 common gene clusters. The largest number of total gene clusters (63) was found when comparing genes in strains of B. melitensis C-573 and B. melitensis ATCC 23457. For B. melitensis C-573 strain, we have found two unique clusters containing two genes each. The first cluster contains genes encoding hypothetical proteins, second- genes of signal peptidase. All genes included in the unique clusters are located on the first chromosome of the genome of $B$. melitensis strain C-573.

\subsection{Assessment of gene expression level}

To assess the gene expression level, we have used the method of whole transcriptome sequencing. The reads obtained as a result of the sequencing were filtered and mapped to the genome. Assessment of gene expression level was performed as described in Materials and Methods section. In order to normalize the results of the mapping, we have evaluated RPKM (Reads per kilobase per million mapped reads) value for each gene. Average RPKM value was 117.9; maximum and minimum were 266.6 and 0, respectively. Results of the gene expression level assessment are presented in Table S2 «Gene expression level».

100 genes with maximum gene expression level were assigned to functional COG categories, among them for, $37 \%$ of genes function is unknown; 9\% belong to amino acid transport and metabolism cluster; $9 \%$ take part in the process of 
replication; $6 \%$ are in transcription processes; $4 \%$ are genes encoding proteins of carbohydrate transport and metabolism; $3 \%$ encode proteins with general function. The least represented were the functional categories of genes involved in the production and conversion of energy, lipid metabolism, translation, ion transport and metabolism-2\% of genes for each category. The number of genes belonging to the functional categories of cell cycle control, coenzyme metabolism, cell wall biogenesis, post-translational modification and the secondary structure was $1 \%$ for each of these categories. For $15 \%$ of the genes, the functional category was not assigned. Detailed information on genes with the highest expression level is given in Table 4.

Table 4. Genes with the highest expression level

\begin{tabular}{|c|c|c|c|}
\hline Locus tag & COG category & RPKM & Protein name \\
\hline BMEI1814 & $\mathrm{L}$ & 266.6 & transposase \\
\hline BMEI1815 & $\mathrm{L}$ & 238.7 & transposase \\
\hline BMEI1897 & $\mathrm{S}$ & 230.3 & hypothetical protein \\
\hline BMEI1656 & $\mathrm{S}$ & 217.7 & hypothetical protein \\
\hline BMEI0848 & $\mathrm{S}$ & 216.5 & probable carnitine operon oxidoreductase caia \\
\hline BMEI1658 & $\mathrm{S}$ & 215.9 & hypothetical protein \\
\hline BMEI1405 & $\mathrm{L}$ & 215.1 & transposase \\
\hline BMEI0142 & $\mathrm{S}$ & 214.0 & hypothetical membrane spanning protein \\
\hline BMEI1000 & $\mathrm{S}$ & 213.5 & hypothetical protein \\
\hline BMEI0141 & $\mathrm{C}$ & 212.2 & dihydrolipoamide succinyltransferase component (e2) of 2-oxoglutarate dehydrogenase complex \\
\hline BMEI1413 & M & 211.7 & gdp-mannose 4,6-dehydratase \\
\hline BMEI0570 & $\mathrm{S}$ & 211.5 & hypothetical protein \\
\hline BMEI0059 & $\mathrm{S}$ & 211.1 & hypothetical protein \\
\hline BMEI1896 & $\mathrm{S}$ & 210.7 & hypothetical membrane spanning protein \\
\hline BMEI1386 & G & 210.6 & xylulose kinase \\
\hline BMEI0585 & $\mathrm{D}$ & 210.5 & cell division protein $\mathrm{ftsz}$ \\
\hline BMEI1738 & $\mathrm{K}$ & 210.0 & transcriptional activator tena \\
\hline BMEI0900 & $\mathrm{S}$ & 209.9 & hypothetical protein \\
\hline BMEII1042 & $\mathrm{S}$ & 209.9 & hypothetical protein \\
\hline BMEI1687 & not in COG & 209.9 & hypothetical protein \\
\hline BMEI1402 & $\mathrm{L}$ & 209.8 & transposase \\
\hline BMEII0912 & $\mathrm{S}$ & 209.6 & hypothetical protein \\
\hline BMEI0058 & $\mathrm{S}$ & 208.2 & extracellular serine protease \\
\hline BMEI0541 & $\mathrm{S}$ & 207.6 & hypothetical protein \\
\hline BMEI1054 & $\mathrm{J}$ & 207.2 & bacterial peptide chain release factor 2 (rf-2) \\
\hline BMEII0182 & $\mathrm{S}$ & 207.0 & hypothetical protein \\
\hline BMEII0470 & not in $\mathrm{COG}$ & 206.9 & integral membrane protein \\
\hline BMEI0994 & $\mathrm{S}$ & 206.4 & hypothetical protein \\
\hline BMEI1657 & $\mathrm{S}$ & 206.0 & hypothetical protein \\
\hline BMEI1686 & $\mathrm{S}$ & 205.9 & hypothetical protein \\
\hline BMEI1680 & $\mathrm{S}$ & 205.7 & hypothetical protein \\
\hline BMEI0389 & S & 204.9 & hypothetical protein \\
\hline BMEI1664 & $\mathrm{L}$ & 204.6 & hypothetical cytosolic protein \\
\hline BMEI1306 & $\mathrm{S}$ & 204.4 & porin \\
\hline BMEII0044 & not in COG & 204.3 & hypothetical protein \\
\hline BMEI0847 & not in COG & 204.0 & hypothetical protein \\
\hline BMEII0559 & $\mathrm{E}$ & 203.7 & aminomethyltransferase \\
\hline BMEI0451 & not in COG & 203.6 & 2-isopropylmalate synthase \\
\hline BMEI0999 & $\mathrm{S}$ & 203.5 & hypothetical protein \\
\hline BMEI0133 & $\mathrm{E}$ & 203.4 & diaminopimelate epimerase \\
\hline BMEI1663 & $\mathrm{K}$ & 203.4 & antirepressor protein ant \\
\hline BMEI0060 & $\mathrm{S}$ & 203.3 & hypothetical protein \\
\hline BMEII1070 & $\mathrm{S}$ & 203.0 & adhesin aida-i \\
\hline BMEI0778 & $\mathrm{F}$ & 202.9 & adenylate kinase \\
\hline BMEI1678 & $\mathrm{S}$ & 202.8 & hypothetical protein \\
\hline BMEI1881 & S & 202.4 & hypothetical protein \\
\hline
\end{tabular}




\begin{tabular}{|c|c|c|}
\hline BMEI0496 & $\mathrm{R}$ & 201.8 \\
\hline BMEI1542 & $\mathrm{L}$ & 201.7 \\
\hline BMEII0878 & K & 201.7 \\
\hline BMEI1397 & $\mathrm{L}$ & 201.1 \\
\hline BMEI0827 & I & 201.0 \\
\hline BMEII0560 & E & 200.8 \\
\hline BMEII0919 & S & 200.5 \\
\hline BMEI1700 & K & 200.5 \\
\hline BMEI0993 & S & 200.4 \\
\hline BMEI1699 & not in $\mathrm{COG}$ & 200.1 \\
\hline BMEI0198 & not in $\mathrm{COG}$ & 199.9 \\
\hline BMEII0911 & E & 199.8 \\
\hline BMEII0483 & G & 199.5 \\
\hline BMEII0247 & $\mathrm{R}$ & 199.3 \\
\hline BMEI1696 & not in COG & 199.3 \\
\hline BMEI1416 & $\mathrm{G}, \mathrm{M}$ & 199.3 \\
\hline BMEII0482 & S & 199.2 \\
\hline BMEI1684 & S & 199.1 \\
\hline BMEII0973 & $\mathrm{C}$ & 199.0 \\
\hline BMEI1674 & S & 199.0 \\
\hline BMEII1133 & E & 198.9 \\
\hline BMEI1486 & $\mathrm{O}$ & 198.8 \\
\hline BMEII1046 & S & 198.7 \\
\hline BMEII0853 & $\mathrm{T}, \mathrm{K}$ & 198.5 \\
\hline BMEI0434 & E & 198.4 \\
\hline BMEI0081 & $\mathrm{H}$ & 198.4 \\
\hline BMEII0902 & K & 198.1 \\
\hline BMEI1916 & F & 198.1 \\
\hline BMEI1813 & S & 198.0 \\
\hline BMEI0052 & $\mathrm{S}$ & 198.0 \\
\hline BMEII0706 & $\mathrm{R}$ & 197.9 \\
\hline BMEII0571 & E & 197.9 \\
\hline BMEI0022 & I & 197.8 \\
\hline BMEII0480 & not in $\mathrm{COG}$ & 197.7 \\
\hline BMEI1775 & $\mathrm{J}$ & 197.3 \\
\hline BMEI2024 & $\mathrm{L}$ & 197.2 \\
\hline BMEI0546 & Q & 197.2 \\
\hline BMEI1690 & not in $\mathrm{COG}$ & 197.1 \\
\hline BMEII0100 & E & 197.1 \\
\hline BMEI1307 & $\mathrm{L}$ & 196.9 \\
\hline BMEII0439 & $\mathrm{P}$ & 196.7 \\
\hline BMEII0478 & G & 196.6 \\
\hline BMEI0375 & $\mathrm{P}$ & 196.5 \\
\hline BMEI0907 & S & 196.5 \\
\hline BMEII0892 & not in $\mathrm{COG}$ & 196.0 \\
\hline BMEI0263 & E & 195.8 \\
\hline BMEI0539 & not in $\mathrm{COG}$ & 195.6 \\
\hline BMEI0996 & not in COG & 195.6 \\
\hline BMEII1092 & G & 195.2 \\
\hline BMEI0633 & not in $\mathrm{COG}$ & 195.2 \\
\hline BMEI1880 & S & 195.0 \\
\hline BMEII0785 & K & 195.0 \\
\hline BMEI1220 & not in $\mathrm{COG}$ & 195.0 \\
\hline BMEI0502 & $\mathrm{S}$ & 194.7 \\
\hline
\end{tabular}

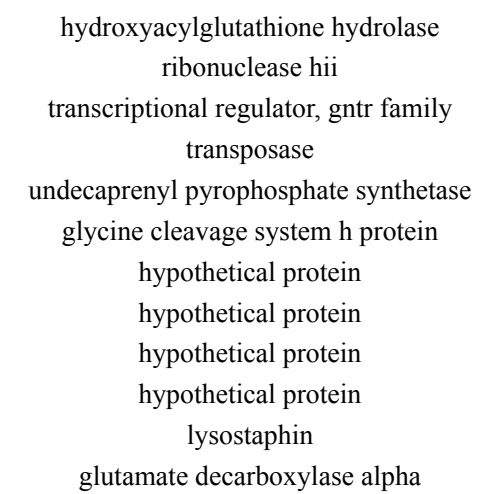

$\mathrm{ABC}$ transporter integral membrane protein exoenzymes regulatory protein aepa precursor

hypothetical membrane spanning protein

o-antigen export system ATP-binding protein rfbb

hypothetical protein

hypothetical protein

nitrous-oxide reductase

hypothetical protein

ornithine decarboxylase

DNA repair protein rada

hypothetical protein

two component response regulator

periplasmic dipeptide transport protein precursor methyltransferase

transcriptional regulatory protein, lysr family cytidylate kinase

hypothetical protein

hypothetical protein

hypothetical cytosolic protein

acetolactate synthase IolD

3-hydroxybutyryl-CoA dehydratase hypothetical protein

ribonuclease ph

hypothetical cytosolic protein

pyrazinamidase/nicotinamidase hypothetical protein

high-affinity branched-chain amino acid transport ATP-binding protein livg integrase

transporter, dme family

d-mannonate oxidoreductase

ferric uptake regulation protein

hypothetical protein

hypothetical protein

hypothetical protein

hypothetical protein

hypothetical protein

hydroxypyruvate isomerase

integral membrane protein

hypothetical membrane spanning protein

transcriptional regulator, marr family

hypothetical protein

hypothetical membrane spanning protein

One-letter abbreviations for the functional categories: C-energy production and conversion; D-cell division and chromosome partitioning; E-amino acid metabolism and transport; F-nucleotide metabolism and transport; G-carbohydrate metabolism and transport; H-coenzyme metabolism; I-lipid metabolism; J-translation, including ribosome structure and biogenesis; K-transcription; L-replication, recombination and repair; M-cell wall structure and biogenesis and outer membrane; O-molecular chaperones and related functions; P-inorganic ion transport and metabolism; Q-Secondary metabolites biosynthesis, transport, and catabolism; S-no functional prediction. R-general functional prediction only; T-signal transduction. 
It is a known fact that the Brucella spp. do not have common bacterial virulence factors, such as cytolysins, exotoxins, secreted proteases, fimbriae, phage-encoded toxins, and virulence plasmids ${ }^{[9]}$. Brucella virulence is determined by their ability to survive and reproduce in the macrophages of the host organism. Nowadays factors that are crucial for ensuring the intracellular processes of Brucella cells and affecting their survival inside macrophages have been identified; these include lipopolysaccharide (LPS), brucebactin which is involved in the iron uptake, cyclic $\beta$-1,2-glucans (C $\beta \mathrm{G})$, type IV secretion system and a two-component BvrR/BvrS regulation system ${ }^{[20-25]}$. Although these virulence factors are only indirectly associated with the clinical manifestations of brucellosis, they are crucial for the survival and reproduction of the Brucella cells within host cells.

We have performed gene expression level analysis for genes associated with virulence via comparing median values for each gene group. The results of evaluating the level of expression of B. melitensis C-573 genes associated with virulence are shown in Table 5.

Table 5. The expression level of $\boldsymbol{B}$. melitensis $\mathrm{C}-573$ genes associated with virulence

\begin{tabular}{|c|c|c|c|c|}
\hline Gene & Symbol & RPKM & Virulence factor & Protein \\
\hline BMEII0076 & vibH/entF & 178 & Brucebactin & enterobactin synthetase component $\mathrm{F}$ \\
\hline BMEII0077 & dhbC & 180.4 & Brucebactin & isochorismate synthase \\
\hline BMEII0078 & dhbE & 48.1 & Brucebactin & 2,3-dihydroxybenzoate-amp ligase \\
\hline BMEII0079 & dhbB & 34.9 & Brucebactin & isochorismatase \\
\hline BMEII0080 & dhbA & 42.5 & Brucebactin & 2,3-dihydroxybenzoate-2,3-dehydrogenase \\
\hline BMEII0081 & entD & 44.2 & Brucebactin & enterobactin syntetase component D \\
\hline BMEI2035 & bvrS & 18.7 & BvrR/BvrS & sensor protein BvrS \\
\hline BMEI2036 & bvrR & 4.7 & BvrR/BvrS & transcriptional regulatory protein BvrR \\
\hline BMEI1837 & cgs & 159.2 & $\mathrm{C} \beta \mathrm{G}$ & cyclic beta 1-2 glucan synthetase \\
\hline BMEI0509 & $1 \mathrm{psB} / \mathrm{lpcC}$ & 129.6 & LPS & lipopolysaccharide core biosynthesis mannosyltransferase LpcC \\
\hline BMEI0586 & $\operatorname{lpxC}$ & 120.5 & LPS & UDP-3-O-[3-hydroxymyristoyl] N-acetylglucosamine deacetylase \\
\hline BMEI0831 & $1 \mathrm{pxD}$ & 137.5 & LPS & UDP-3-O-[3-hydroxymyristoyl] glucosamine N-acyltransferase \\
\hline BMEI0832 & fabZ & 130 & LPS & (3R)-hydroxymyristoyl ACP dehydratase \\
\hline BMEI0833 & $\operatorname{lpxA}$ & 126 & LPS & UDP-N-acetylglucosamine acyltransferase \\
\hline BMEI0835 & $\operatorname{lpxB}$ & 125.6 & LPS & lipid-A-disaccharide synthase \\
\hline BMEI0850 & kdsA & 98.4 & LPS & 2-dehydro-3-deoxyphosphooctonate aldolase \\
\hline BMEI0997 & wbdA & 179.2 & LPS & mannosyltransferase \\
\hline BMEI0998 & wboA & 176.7 & LPS & glycosyltransferase \\
\hline BMEI1111 & acpXL & 174.1 & LPS & acyl carrier protein \\
\hline BMEI1115 & htrB & 78.2 & LPS & lipid A biosynthesis lauroyl acyltransferase \\
\hline BMEI1212 & $\operatorname{lpxE}$ & 131.9 & LPS & phosphatidylglycerophosphatase B \\
\hline BMEI1326 & $\operatorname{lps} \mathrm{A}$ & 144.8 & LPS & glycosyltransferase \\
\hline BMEI1393 & wbpZ & 180.4 & LPS & mannosyltransferase $\mathrm{C}$ \\
\hline BMEI1394 & manAoAg & 157.3 & LPS & mannose-6-phosphate isomerase \\
\hline BMEI1395 & $\operatorname{manCoAg}$ & 147.6 & LPS & mannose-1-phosphate guanylyltransferase \\
\hline BMEI1396 & $\mathrm{pmm}$ & 183.8 & LPS & phosphomannomutase \\
\hline BMEI1404 & wbkA & 193.5 & LPS & mannosyltransferase \\
\hline BMEI1413 & gmd & 211.7 & LPS & GDP-mannose 4,6-dehydratase \\
\hline BMEI1414 & per & 181.3 & LPS & perosamine synthetase \\
\hline BMEI1415 & wzm & 189.3 & LPS & O-antigen export system permease protein \\
\hline BMEI1416 & wzt & 199.3 & LPS & O-antigen export system ATP-binding protein \\
\hline BMEI1417 & wbkB & 185.8 & LPS & perosamine synthetase $\mathrm{WbkB}$ \\
\hline BMEI1418 & wbkC & 170 & LPS & $\begin{array}{c}\text { GDP-mannose 4,6-dehydratase/GDP-4-amino-4,6-dideoxy-D-mannose } \\
\text { formyltransferase }\end{array}$ \\
\hline BMEI1426 & wbpL & 186.1 & LPS & undecaprenyl-phosphate alpha-N-acetylglucosaminyltransferase \\
\hline BMEI1475 & acpXL & 164.2 & LPS & acyl carrier protein \\
\hline BMEI1886 & pgm & 171.9 & LPS & phosphoglucomutase \\
\hline
\end{tabular}




$\begin{array}{ccccc}\text { BMEI1904 } & \text { kdsB } & 169.5 & \text { LPS } & \begin{array}{c}\text { 3-deoxy-manno-octulosonate cytidylyltransferase } \\ \text { phosphomannomutase }\end{array} \\ \text { BMEII0899 } & \text { manBcore } & 146.9 & \text { LPS } & \begin{array}{c}\text { mannose-6-phosphate isomerase/mannose-1-phosphate guanylyltransferase (GDP) } \\ \text { tetraacyldisaccharide 4'-kinase }\end{array} \\ \text { BMEII0900 } & \text { manCcore } & 157.1 & \text { LPS } & \text { 3-deoxy-D-manno-octulosonic-acid transferase } \\ \text { BMEII1029 } & \text { lpxK } & 37.9 & \text { LPS } & \text { lytic transglycosylase } \\ \text { BMEII0025 } & \text { virB1 } & 164.4 & \text { T4SS } & \text { Pilus } \\ \text { BMEII0026 } & \text { virB2 } & 183.3 & \text { T4SS } & \text { channel protein } \\ \text { BMEII0027 } & \text { virB3 } & 156.2 & \text { T4SS } & \text { ATPase } \\ \text { BMEII0028 } & \text { virB4 } & 173.4 & \text { T4SS } & \text { Attachment mediating protein } \\ \text { BMEII0029 } & \text { virB5 } & 177.5 & \text { T4SS } & \text { IM Channel protein } \\ \text { BMEII0030 } & \text { virB6 } & 182.9 & \text { T4SS } & \text { channel protein } \\ \text { BMEII0031 } & \text { virB7 } & 185.4 & \text { T4SS } & \text { channel protein } \\ \text { BMEII0032 } & \text { virB8 } & 189.5 & \text { T4SS } & \text { channel protein } \\ \text { BMEII0033 } & \text { virB9 } & 190.7 & \text { T4SS } & \text { ATPase } \\ \text { BMEII0034 } & \text { virB10 } & 171.6 & \text { T4SS } & \text { outer membrane protein } \\ \text { BMEII0035 } & \text { virB11 } & 167.8 & \text { T4SS } & \text { T4SS } \\ \text { BMEII0036 } & \text { virB12 } & 183.7 & & \end{array}$

For genes involved in the iron uptake RPKM value is 46 , for $\mathrm{C} \beta \mathrm{G}$ gene-159.2; for genes of $\mathrm{BvrR} / \mathrm{BvrS}$ regulation system-11.35; for T4SS genes-179.5; for LPS group-170. Low level of gene expression for BvrR/BvrS regulation system and iron uptake genes may be explained by the fact that nutrient medium provided comfortable conditions for the growth of the $B$. melitensis $\mathrm{C}-573$ culture, such as the absence of stress factors at low $\mathrm{pH}$ value, presence of antibiotics, low iron content. The expression level of genes associated with virulence factors T4SS and LPS significantly exceeds average RPKM values estimated for all other B. melitensis $\mathrm{C}-573$ genes, which indicates that the protein products of these genes play a great role not only in successful survival in the host organism, but also in survival on a nutrient medium.

\section{Conclusion}

The results of our study expand the current understanding of the structural and functional organization of the $B$. melitensis genome. This is the first analysis of the completed genome of a clinical B. melitensis strain isolated in Russia. The created genomic project is a valuable reference material for future studies of the virulence of the pathogen. The genomic data we obtained in combination with the genetic data of other virulent and vaccine strains can make a significant contribution to the study of the pathogenicity of Brucella and the development of effective therapeutic and preventative means for controlling brucellosis.

\section{Funding}

No funding was received for this research.

\section{Conflict of interest}

The authors declare no conflicts of interest.

\section{Acknowledgments}

Not applicable

This article contains supplementary information online at: http://ojs.wiserpub.com/index.php/AMTT/article/view/626/407

\section{References}

[1] P. de Figueiredo, T. A. Ficht, A. Rice-Ficht, C. A. Rossetti, L. G. Adams. Pathogenesis and immunobiology of brucellosis: Review of brucellae host interactions. The American Journal of Pathology. 2015; 185(6): 1505-1517. 
[2] A. M. Whatmore, N. Davison, A. Cloeckaert, et al. Brucella papionis sp. nov., isolated from baboons (Papio spp.). International Journal of Systematic and Evolutionary Microbiology. 2014; 64: 4120-4128.

[3] H. C. Scholz, S. Revilla-Fernández, S. Al Dahouk, et al. Brucella vulpis sp. nov., isolated from mandibular lymph nodes of red foxes (Vulpes vulpes). International Journal of Systematic and Evolutionary Microbiology. 2016; 66: 2090-2098.

[4] E. M. Galinska, J. Zagorski. Brucellosis in humans-etiology, diagnostics, clinical forms. Annals of Agricultural and Environmental Medicine. 2013; 20: 233-238.

[5] B. Mantur, S. Amarnath, R. Shinde. Review of clinical and laboratory features of human Brucellosis. Indian Journal of Medical Microbiology. 2007; 25: 188-202.

[6] G. Pappas, P. Papadimitriou, N. Akritidis, L. Christou, E. V. Tsianos. The new global map of human brucellosis. The Lancet Infectious Diseases. 2006; 6: 91-99.

[7] K. A. Franc, R. C. Krecek, B. N. Häsler, A. M. Arenas-Gamboa. Brucellosis remains a neglected disease in the developing world: a call for interdisciplinary action. BMC Public Health. 2018; 18: 125.

[8] D. G. Ponomarenko, D. V. Rusanova, T. V. Berdnikova, A. A. Khachaturova, E. A. Manin, A. N. Kulichenko. Overview of epizootological and epidemiological situation on Brucellosis in the Russian federation in 2017 and prognosis for 2018. Problemy Osobo Opasnykh Infektsii. 2018; 2: 23-29.

[9] V. G. DelVecchio, V. Kapatral, R. J. Redkar, et al. The genome sequence of the facultative intracellular pathogen Brucella melitensis. Proceedings of the National Academy of Sciences of the United States of America. 2002; 99: 443448.

[10] S. V. Pisarenko, D. A. Kovalev, A. S. Volynkina, et al. Global evolution and phylogeography of Brucella melitensis strains. BMC Genomics. 2018; 19(1): 353.

[11] S. Andrews. FastQC: a quality control tool for high throughput sequence data. 2010. Available from: http://www. bioinformatics.babraham.ac.uk/projects/fastqc.

[12] A. M. Bolger, M. Lohse, B. Usadel. Trimmomatic: A flexible trimmer for Illumina sequence data. BioInformatics. 2014; 30: 2114-2120.

[13] A. C. Darling, B. Mau, F. R. Blattner, N. T. Perna. Mauve: multiple alignment of conserved genomic sequence with rearrangements. Genome Research. 2004; 14(7): 1394-1403.

[14] G. Lu, E. N. Moriyama. Vector NTI, a balanced all-in-one sequence analysis suite. Briefings in bioinformatics. 2004; 5(4): 378-88.

[15] National Center for Biotechnology Information Search Database. Available from: https://www.ncbi.nlm.nih.gov/ [Accessed 20th November 2020].

[16] Y. Wang, D. Coleman-Derr, G. Chen, Y. Q. Gu. OrthoVenn: A web server for genome wide comparison and annotation of orthologous clusters across multiple species. Nucleic Acids Research. 2015; 43(W1):W78-84.

[17] D. Kim, B. Langmead, S. L. Salzberg, HISAT: A fast spliced aligner with low memory requirements. Nature Methods. 2015; 12(4): 357-60.

[18] S. Anders, P. T. Pyl, W. Huber HTSeq-A python framework to work with high-throughput sequencing data. Bioinformatics. 2015; 31(2): 166-169.

[19] A. Mortazavi, B. A. Williams, K. McCue, L. Schaeffer, B. Wold. Mapping and quantifying mammalian transcriptomes by RNA-Seq. Nature Methods. 2008; 5(7): 621-628.

[20] Z. Xiang, W. Zheng, Y. He. BBP: Brucella genome annotation with literature mining and curation. BMC Bioinformatics. 2006; 7: 347.

[21] N. Lapaque, I. Moriyon, E. Moreno, J. P. Gorvel. Brucella lipopolysaccharide acts as a virulence factor. Current Opinion in Microbiology. 2005; 8: 60-66.

[22] L. Eskra, J. Covert, J. Glasner, G. Splitter. Differential expression of iron acquisition fenes by Brucella melitensis and Brucella canis during macrophage infection. PLoS One. 2012; 7(3): e31747.

[23] B. Arellano-Reynoso, N. Lapaque, S. Salcedo, et al. Cyclic $\beta-1,2$-glucan is a Brucella virulence factor required for intracellular survival. Nature Immunology. 2005; 6: 618-625.

[24] D. O'Callaghan, C. Cazevieille, A. Allardet-Servent, et al. A homologue of the Agrobacterium tumefaciens VirB and Bordetella pertussis Ptl type IV secretion systems is essential for intracellular survival of Brucella suis. Molecular Microbiology. 1999; 33: 1210-1220.

[25] C. Guzman-Verri, L. Manterola, A. Sola-Landa, et al. The two-component system BvrR/BvrS essential for Brucella abortus virulence regulates the expression of outer membrane proteins with counterparts in members of the Rhizobiaceae. Proceedings of the National Academy of Sciences of the United States of America. 2002; 99: 1237512380. 